\title{
Retos del área de talento humano frente a la inserción laboral de personas en proceso de reintegración ${ }^{9}$
}

\author{
Diana Carolina Maya Cabrera \\ Psicóloga \\ Universidad de Nariño \\ Pasto. Colombia \\ Correo electrónico: dianacmayac@gmail.com
}

\section{Yanitza Nicole Flórez Rodríguez \\ Psicóloga \\ Universidad de Nariño \\ Pasto. Colombia \\ Correo electrónico: nicole.florez.r@gmail.com}

\section{Sonia Maritza Matabanchoy Tulcán \\ Ph.D Psicología Universidad de Nariño \\ Pasto. Colombia \\ Correo electrónico: somapsicologa@yahoo.es}

\section{Resumen}

Colombia lleva a cabo un proceso de reintegración para los excombatientes, a fin de mejorar su calidad de vida a través de diferentes oportunidades, entre ellas, en el ámbito laboral, generando procesos de inserción en las organizaciones. Sin embargo, es necesario que esta apertura sea completa, a través de cambios sustanciales dentro del área de talento humano. Por esto, la presente investigación tiene como objetivo reconocer los retos del área de Talento Humano frente a la inserción laboral de personas en proceso de reintegración en la ciudad de San Juan de Pasto, a partir del enfoque cualitativo de tipo fenomenológico, con la participación de cinco jefes de talento humano del sector de construcción. Como resultados se encontraron cinco categorías inductivas para responder a los objetivos. Se encontró que existe apertura a la inserción laboral dentro de las empresas, además del reconocimiento y la gestión de cambio en los procesos de talento humano.

\section{Palabras clave}

Talento humano, inserción laboral, proceso de reintegración, empresas, Colombia, retos.

9 Para citar este artículo: Maya, D. Flórez, N. Y., \& Matabanchoy, S. (2019). Retos del área de talento humano frente a la inserción laboral de personas en proceso de reintegración. Una revisión. Informes Psicológicos, 19(1), pp. 163-180 http://dx.doi.org/10.18566/infpsic.v19n1a09 


\title{
Challenges of the human talent area in the face of the employability of people in the process of reintegration
}

\begin{abstract}
Colombia carries out a process of reintegration for ex-combatants, in order to improve their quality of life through different opportunities, for instance in the workplace, through the generation of insertion processes in the organizations. However, it is necessary that this opening be complete, and that substantial changes within the human talent area be applied. This research aims to recognize the challenges of the human talent area against the employability of people who are in the process of reintegration in the city of San Juan de Pasto. The study is based on the qualitative approach of phenomenological type, and it counts on the participation of five human talent heads from the construction sector. Five inductive categories were found to respond to the objectives. As a result, it was found that there is openness to labor insertion within the companies, in addition to the recognition and change management in human talent processes.
\end{abstract}

Keywords

Human talent, labor insertion, employability, reintegration process, companies, Colombia, challenges.

\section{Desafios da área de talentos humanos diante da inserção laboral de pessoas em processo de reintegração}

\section{Resumo}

A Colômbia realiza um processo de reintegração de ex-combatentes, com o objetivo de melhorar sua qualidade de vida por meio de diferentes oportunidades, inclusive no ambiente de trabalho, gerando processos de inserção nas organizações. No entanto, é necessário que esta abertura seja completa, através de mudanças substanciais dentro da área do talento humano. Portanto, esta pesquisa visa reconhecer os desafios da área do Talento Humano frente à inserção laboral de pessoas no processo de reintegração na cidade de San Juan de Pasto, a partir da abordagem qualitativa do tipo fenomenológico, com a participação de cinco chefes de talentos humanos do setor de construção. Como resultados, cinco categorias indutivas foram encontradas para responder aos objetivos. Verificou-se que existe abertura para inserção laboral nas empresas, além do reconhecimento e gestão de mudanças nos processos de talentos humanos.

Palavras chave

Talento humano, inserção laboral, processo de reintegração, empresas, Colômbia, desafios. 


\section{ntroducción}

Desde aproximadamente seis décadas Colombia se enfrenta al conflicto armado, el cual ha desencadenado hechos dolorosos para la población civil; uno de los principales grupos implicados en la guerra fue las Fuerzas Armadas Revolucionarias de Colombia (FARC), que surgió en respuesta a exclusiones principalmente, sociales y políticas, del Estado en diferentes ámbitos del país (López, Silva, Castro, \& Caicedo, 2016).

En el transcurso de la guerra se han visto afectadas diferentes zonas y poblaciones del país, encontrando como noticias habituales hechos victimizantes de reclutamiento forzado, minas antipersonales, desplazamiento interno, secuestros, violencia física, psicológica y sexual; por ello, la comunidad ha asumido un papel activo dentro de los procesos que se adelanten referentes a la construcción de paz.

Se evidencia entonces que las víctimas y las personas en proceso de reintegración juegan un papel predominante en las esferas donde se hallan inmersos, como el campo económico, social, histórico, cultural, familiar, político, etc., campos que fueron revestidos de sufrimiento, dolor y pérdida, y que ha traído consigo estancamiento individual y colectivo. Por estas razones, se puede esperar rechazo por parte de la comunidad hacia los perpetradores de violencia, con o sin el conocimiento suficiente para tomar una actitud desfavorable hacia ellos (López et al., 2016), hasta el punto de presentar algún tipo estigmatización, dificultando su inclusión dentro de la comunidad civil.
No obstante, el conflicto y la guerra fueron vistos de una manera distinta por los grupos guerrilleros, puesto que ellos relacionaban el conflicto armado como una lucha que permitiría la transformación estructural de la sociedad, para que así, las poblaciones menos favorecidas pudieran gozar de los derechos de unos cuantos. Sin embargo, esta lucha se tergiversó por años, convirtiéndose en actos terroristas y delictivos que afectaron toda la población colombiana (Torres et al., 2016).

Es por esto que el Estado Colombiano en el año 2012, asumió negociaciones para dar cese al conflicto con las FARC, desde entonces, se implementa el proceso de desmovilización, y consecuente reintegración social, en el que los integrantes de este grupo armado tienen la posibilidad de vincularse y adaptarse a la sociedad civil, a través de las estrategias que brinda el gobierno (Roldán, 2013). Dicha reintegración, es uno de los retos fundamentales del posconflicto, entendida por las Naciones Unidas como, un tipo de adopción de medidas orientadas a fortalecer el potencial personal, social, educativo, socioeconómico de los ex combatientes y de sus familias. La ayuda puede ser de forma monetaria, compensación en especie, entrenamiento vocacional, estudios y enseñanzas de un oficio y programas de empleo (Martínez, Ocampo, Betancur, \& Bonilla, 2015).

Es por eso que la Agencia para la Reincorporación y la Normalización (ARN) ha liderado este proceso, puesto que brinda acogida y busca potenciar los talentos, las capacidades y habilidades que posee cada persona desmovilizada. Ante esto, se crea la Ruta de Reintegración, donde se trabajan ocho 
dimensiones, una de ellas es la dimensión productiva, en la que se centra la presente investigación. En ésta se busca la generación de capacidades que faciliten la inserción económica de las personas en proceso de Reintegración, de manera que, tanto ellas como sus grupos familiares, puedan disfrutar de sus derechos económicos y desarrollar un proyecto de vida productivo, según sus expectativas y el entorno en el que se encuentran, dentro del marco legal (ARN, 2016).

Dentro de la dimensión productiva se desarrollan cinco componentes: oportunidades del contexto económico, fortalecimiento del talento, generación de ingresos, opciones productivas, familia y manejo financiero; todas ellas encaminadas a fortalecer las capacidades de las personas en proceso de reintegración y su grupo familiar para la producción de ingresos sostenibles en el marco de la legalidad, que sean acorde con sus potencialidades y el contexto económico (ARN, 2016).

El objetivo de consolidar la paz y el Estado social de derecho a largo plazo, en Colombia necesariamente implicará un gran consenso social en el que participen no sólo grupos sociales, instituciones, gobernantes y ciudadanos, sino también las empresas y otros actores privados que ejercen un rol determinante dentro de esta visión (Fundación Ideas para la Paz, FIP, 2017)

Se conoce entonces que el sector privado cuenta con capacidades técnicas, administrativas, organizacionales, financieras y capital humano para contribuir con la inserción laboral de personas en proceso de reintegración. Más allá de estas capacidades, su aporte no se limita a la creación de empleo y oportunidades económicas, todo constituye un conjunto de acciones que las empresas pueden lograr en este proceso, en que el fin último está en contribuir a la construcción de paz y bienestar (FIP, 2017)

Desde la experiencia que ha llevado a cabo la ARN con relación a la empresa privada, se ha conseguido resultados positivos, en donde más de 2000 personas se han beneficiado de voluntariados o capacitaciones en temas relacionados con la creación de negocio y el emprendimiento. Por esta razón, la ARN se ha convertido en un aliado fundamental del sector privado en el proceso de reintegración (Cabrera \& Niño, 2016).

También, en la dimensión productiva, se aborda la inserción laboral, entendida como la posibilidad que se brinda a la población para acceder a una oportunidad laboral formal o informal con un pago que le permita solventar las necesidades fundamentales. Estos procesos de inserción implican que esta población sea un ente activo dentro del tejido social del país, mejorando su calidad de vida, y contribuyendo al desarrollo de la organización (Jiménez, 2013).

La Responsabilidad Social Empresarial (RSE) o la Responsabilidad Social Corporativa (RSC) va más allá que sólo generar empleo; en esta se hace necesario conocer y transformar la actitud, el pensamiento y el actuar de la empresa en relación con el proceso de paz para así dar ejemplo a la sociedad y que ésta sea consciente de la reintegración como un proceso necesario para el mejoramiento del país (Martínez et al., 2015). 
Al comprender la importancia de la dimensión productiva y la inserción laboral, el área de talento humano juega un papel importante en el proceso de reintegración dentro de las organizaciones, pues es el encargado de gestionar el talento humano de una organización por medio de los procesos de reclutamiento, selección, capacitación, evaluación del desempeño, seguridad y salud en el trabajo , clima organizacional y bienestar laboral, etc., buscando un mejoramiento continuo de la empresa, además de un éxito económico y humano, a partir de una orientación consciente y pragmática, dirigida a desarrollar y fortalecer las potencialidades, capacidades, conocimientos y destrezas en el trabajador (Mejía, Bravo \& Montoya, 2013).

Es aquí otro de los aspectos donde el área de Talento Humano es protagonista, puesto que debe generar, a través de la concientización, el mejoramiento del clima organizacional y la apertura a la construcción de país, desarrollando oportunidades para que cada una de estas personas se reintegre de forma idónea al ámbito laboral y pueda desempeñarse con éxito, gozando de una adecuada adaptación a la cultura organizacional, hecho que facilita la fidelización del colaborador, a la vez que está estrechamente vinculado con el clima laboral, y la productividad de la empresa. Es así como muchas personas en proceso de reintegración pueden apoyar al crecimiento de la empresa, a partir de sus talentos y sus aportes personales (Moreno \& Godoy, 2012). El papel de las organizaciones es de vital importancia a la hora de construir y reconstruir paz en el país, no sólo con aportaciones a nivel económico sino también a nivel social, puesto que las decisiones y acciones de la organización se evidencian en el desarrollo, crecimiento y fortalecimiento del entorno y de los agentes que en éste interactúan a diario (Cabrera \& Niño, 2016).

Por lo planteado hasta el momento, esta investigación se realiza con el objetivo de reconocer los retos del área de talento humano, frente a la inserción laboral de personas en proceso de reintegración, en empresas del sector de construcción, de la ciudad de San Juan de Pasto, para que sea ésta una visión del panorama del sector productivo y la apertura organizacional a las personas en proceso de reintegración en Colombia. Reconociendo los retos a los que se enfrenta el área de Talento humano dentro de este nuevo escenario en el país, se podrán desarrollar estrategias y mecanismos adaptados y adecuados de acuerdo con las necesidades del contexto, que aporten en los diferentes procesos del área de talento humano y contribuyan a la reconstrucción de la sociedad colombiana.

\section{Objetivos específicos de la investigación}

Para reconocer los retos del área de talento humano, frente a la inserción laboral de personas en proceso de reintegración se plantean los objetivos específicos en los que se pretende: a) Develar percepción del jefe de talento humano frente a la inserción laboral de personas en proceso de reintegración en empresas del sector de construcción de la ciudad de San Juan de Pasto; b) Describir los procesos actuales de talento humano frente a la inserción laboral de personas en proceso de reintegración en diferentes empresas del sector de construcción 
en la ciudad de San Juan de Pasto; y, c) Reconocer los retos del área de talento humano frente a la inserción laboral de personas en proceso de reintegración en empresas del sector de construcción de la ciudad de San Juan de Pasto.

Se precisa investigar en el departamento de Nariño pues se encontraron cifras preocupantes con relación a los demás departamentos del país en cuanto a la recepción del Beneficio de Reinserción Económica (BIE), ya que existen 50 unidades de negocio, de las cuales sólo, 38 se encuentran en funcionamiento, que, en comparación con otros departamentos afectados y de acuerdo con la cantidad de personas en proceso de reintegración que el departamento acoge, es una cifra considerablemente baja. Se espera proporcionar bases teóricas que permitan comprender la situación actual y encaminar futuras estrategias de intervención (ARN, 2016).

Por otro lado, el estudio se enfoca en el sector de construcción, pues como lo asegura Stiglitz (2017), la vivienda es un campo que presenta grandes oportunidades para incrementar el desarrollo y la productividad. A su vez, se producen dos beneficios: el primero referido a la creación de empleos para colaboradores de baja y mediana calificación, por su alta demanda de mano de obra y constante flujo de personal. El segundo beneficio es que la vivienda integra la suplencia de necesidades básicas en la vida de una persona. Esto se refleja a diario y tiene una implicación económica, como el incremento en la productividad de la mano de obra. Por ende, asegura que, si se vive mejor, se trabaja mejor, y eso ofrece beneficios para todo el sistema.
También este sector presenta un gran crecimiento en los últimos años: en el 2014 obtuvo un índice de crecimiento de 9.9\% y para el año 2016 el 4.1\%, ubicándose como el segundo sector con mejor desempeño en el país. Dicho crecimiento hace que desde el Estado se presenten programas de inversión que benefician tanto a las empresas de este sector como la población en general (García, 2017).

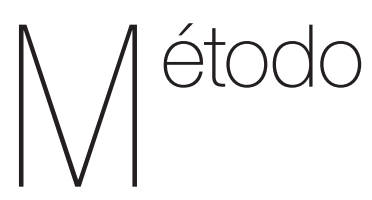

El estudio se realizó bajo el enfoque cualitativo con un tipo de estudio fenomenológico, puesto que permite comprender de manera integral la problemática actual y el contexto del país en el marco del posconflicto. Asimismo, ayudó a develar la percepción de los jefes del área de talento humano frente a la inserción laboral de personas en proceso de reintegración, ya que desde este tipo de estudio se busca comprender el fenómeno a partir de la experiencia propia, desde el relato del individuo, a través de la escucha atenta y la descripción detallada de cada evento.

En cuanto a la unidad de trabajo se contó con la participación de cinco jefes de talento humano del sector de construcción, quienes laboran en diferentes empresas de la ciudad de San Juan de Pasto. La técnica de recolección/producción de información empleada fue la entrevista semiestructurada, la cual contó con la revisión de dos expertas en investigación cualitativa y una experta en el tema a trabajar. La entrevista estuvo conformada por 16 preguntas orientadoras, las cuales estaban organizadas en las tres categorías deductivas, que en la 
aplicación se sustituyeron o ampliaron de acuerdo con el avance en la entrevista.

Los participantes fueron seleccionados cumpliendo con dos criterios: que tuvieran como cargo, ser jefe del área de talento humano y que pertenecieran al sector de construcción dentro de las organizaciones. Así, se contó con la participación de cinco jefes de talento humano. El análisis de información se realizó a través del programa Atlas Ti versión 7, estableciendo categorías deductivas y creando subcategorías inductivas.

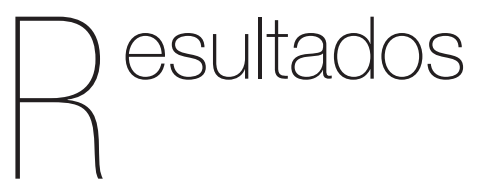

Dentro de los resultados se tuvieron en cuenta las tres categorías de análisis iniciales, a saber: Proceso de reintegración, Inserción laboral y Procesos de talento humano. De las anteriores surgieron tres subcategorías inductivas: Oportunidades de mejora de la inserción laboral, Sector y Retos del área de talento humano.

\section{Proceso de reintegración}

En la categoría Proceso de reintegración los cinco entrevistados evidencian aceptación hacia el proceso de paz, el cual debe llevarse a cabo y continuar su ejecución para así notar cambios sustanciales en la sociedad colombiana. Asimismo, un punto de encuentro en las entrevistas es que, para afianzar la paz en Colombia, cada ciudadano debe contribuir al proceso de reintegración, transformando el pensamiento, la actitud y el actuar de las personas.
También se encuentra, que el proceso de reintegración es visto como una oportunidad de cambio, que propicia desarrollo, crecimiento personal, profesional y laboral; hecho que se convierte en un reto para las empresas, pues se llama a una etapa de superación y cambio de perspectivas que debe ser liderada por los jefes de talento humano.

No obstante, en una de las entrevistas se manifestó que el gobierno debe estructurar de mejor manera este proceso para que en un futuro existan resultados concretos y beneficiosos para esta población y el resto de la sociedad colombiana. Aunado a lo anterior, expresó que era de suma importancia capacitar a la población civil en cuanto al tema, las consecuencias y los aciertos que vendrán en los años siguientes; lo que permitirá un reconocimiento y un cambio de perspectiva acerca del tema de la reintegración.

Es así como se entiende que, por parte de los jefes de talento humano, no existe ningún inconveniente para que estas personas se integren a la sociedad y específicamente al sector productivo, presentando disposición para que la empresa asuma un rol crítico, activo y de apertura frente a este proceso de reintegración, ya que, como se mencionó anteriormente, se desea brindar apoyo a esta población. Sin embargo, uno de los entrevistados expresa incertidumbre frente a la posición que asuman, otros jefes de talento humano frente al proceso, en tanto expresa: "puede ser complicado pues dentro del personal de la empresa, o el mismo jefe de talento humano puede ser víctima de las personas a quien piensa contratar y por eso existirá estigmatización o barreras". 
Por otro lado, se evidencia incertidumbre por el nuevo gobierno en los años siguientes al posconflicto, puesto que se pueden generar cambios drásticos en las políticas en relación con la continuidad del proceso de reintegración, viéndose afectadas las garantías para dicha población y para los colombianos en general.

Por tal razón, se llega a la conclusión de que, ayudando a estos grupos en proceso de desmovilización, también se aporta de manera sustancial al resto de la sociedad. Sin embargo, debe haber una contribución por parte de los ciudadanos para que haya una paz efectiva, ya que estas personas son ciudadanos con los mismos derechos, que desean rehacer su vida a partir de las oportunidades que este proceso les ofrezca, cubriendo con las necesidades básicas de dichas personas y sus familiares.

\section{Inserción laboral}

Dentro de la categoría Inserción laboral se pudo encontrar apertura por parte de estas empresas del sector de construcción para que dichas personas se integren al ámbito laboral; pese a ello, cuatro de las cinco empresas aún no han realizado ningún tipo de contratación con personas en proceso de reintegración. Esto evidencia que, aunque dentro de las empresas exista apertura, no se generan estrategias para reclutar o contratar a esta población, lo que hace que el proceso no avance en cuanto a la generación de empleos para transformar la vida productiva de estas personas y la realidad social. De esta manera, en una de las entrevistas se expone: "la mayoría de empresas de la zona urbana no están preparadas para esto, todos los sectores no están preparados, son grandes retos a los que se enfrenta el país, que se convierten en realidades a las que hay que hacerles frente, hay que hacer cambios, estudiar el tema, para aportar de verdad a la inserción laboral."

Por otra parte, la percepción que tienen los jefes de talento humano acerca de la inserción laboral de estas personas es asertiva, puesto que es vista como una oportunidad para integrarse a la vida laboral y especialmente a la vida en sociedad. Además de que se cree que, si no se brinda este tipo de oportunidades, estas personas volverán a sus antiguas actividades, trayendo consigo daños perjudiciales en la sociedad, como por ejemplo un incremento en el desempleo, la mendicidad y posiblemente delincuencia, así como también afecciones en la vida de los excombatientes como pocas oportunidades para salir adelante, acceder a beneficios del estado y bajos recursos económicos.

Con respecto al aporte de las personas en proceso de reintegración a la empresa, tres de los cinco jefes de talento humano entrevistados consideran que éste no iría más allá de realizar la labor asignada, como lo haría cualquier otro trabajador. Mientras que en las dos siguientes entrevistas, se considera que el aporte de estas personas a la empresa va más allá de cumplir con su labor, pues contribuye a la sensibilización y enseñanza del resto de los trabajadores, por medio de los valiosos testimonios de vida en relación al estar inmersos en la guerra y salir de ésta. También el aporte radica en la disminución de la violencia en la sociedad, y al sector productivo del país por medio de su trabajo como colaboradores en la empresa y creando microempresas 
y empresas que generen empleo para ellos mismos y la población colombiana.

En cuanto al aporte de las empresas sería la contratación, brindando la oportunidad de acceder a un trabajo formal y, en el que se establece una garantía económica tanto para el colaborador como para su familia.

Habría que decir que dentro de las entrevistas se manifestó: la inserción laboral de personas en proceso de reintegración genera estigmas, prejuicios y temor a las personas que conforman una organización. A pesar de que el proceso de paz no es un tema reciente, aún es difícil que la población colombiana acepte que estas personas puedan entrar a un entorno laboral, dado el contexto de donde vienen, puesto que poseen lógicas de trabajo muy diferentes, con reglas y normas que podrían generar conflicto dentro de este nuevo ambiente. Sin embargo, se sabe que para lograr una paz verdadera es necesario dar la apertura a estas personas en el sector productivo liberándose de prejuicios y todo tipo de estigmatización a partir de programas o capacitaciones que concienticen a la comunidad en general, como ya se mencionó anteriormente. Es aquí donde surge una de las categorías inductivas, la cual fue denominada Oportunidades de mejora en la inserción laboral, que se compone de diferentes ideas que surgen dentro de esta problemática.

\section{Oportunidades de mejora frente a la inserción laboral}

Una de las oportunidades de mejora que se encontró dentro de las entrevistas está relacionada con el nivel académico de la mayoría de personas en proceso de reintegración, siendo muy bajo en algunos casos, dificultando la contratación, puesto que sin los conocimientos y la experiencia para cumplir el perfil requerido es imposible contratar a estas personas. En este sentido, en una de las entrevistas se menciona, que la empresa puede brindar oportunidades de estudio a esta población, a través, de cursos, capacitaciones, o brindando las condiciones económicas, de tiempo o accesibilidad a instituciones educativas, que aporten al crecimiento, personal, laboral y profesional del colaborador.

Otro aspecto de mejora que se visualiza dentro de la inserción laboral, es que el clima organizacional puede verse afectado en el momento de integrar a este grupo, ya que muchas personas aún no aceptan ni se adecuan a los cambios realizados por el gobierno en relación al acuerdo de paz y pueden generar algún tipo de discriminación. Que repercuta el clima organizacional, las relaciones interpersonales, la integración, la satisfacción laboral y el desempeño dentro de la empresa; por tal razón, algunos de los directivos de las empresas entrevistadas presentaban mucha incertidumbre en el momento de integrar a esta población sin afectar los procesos que ya se presentan dentro de ésta.

\section{Sector Productivo}

Por otra parte, las personas entrevistadas manifiestan que los reintegrados pueden ocupar cargos operativos en los sectores de construcción, industrial y el campo. Puesto que se considera que la mayoría de personas en proceso de 
reintegración pertenecen a zonas rurales, en las que labores como agricultura, ganadería o piscicultura, se les facilitan, contando que en estas no se requiere de un perfil altamente cualificado, además que en el entorno rural no se establecen protocolos para la contratación, dándose esta frecuentemente de manera informal, u obra labor.

Además, concuerdan en que el sector de construcción puede acoger a personas en proceso de reintegración, puesto que cuenta con cargos donde se pueden desempeñar, los cuales no presentan un perfil riguroso como en otros sectores de las organizaciones y el trabajo. Así como también, dentro de este sector existe un gran flujo de personal, lo que facilita su contratación continua. Se expresa que los colaboradores presentes en este sector, generalmente son de bajos recursos, presentan un alto nivel de vulnerabilidad y gran necesidad de trabajar; realidad que hace que en sector se incrementen los riesgos y accidentes laborales, situación que de alguna forma la legislación en seguridad y salud en el trabajo está tratando de intervenir.

Cabe señalar que dos de los entrevistados mencionaron que en los otros sectores de la industria era más complicado ingresar, ya que existen condiciones y requerimientos específicos para desarrollar la labor y prestar un servicio de calidad; de hacerlo se piensa que accede a un trabajo legal, pero con baja remuneración y garantías laborales. Por ello, es necesario que estas personas se ajusten al perfil que se busca y especialmente a los lineamientos y la plataforma estratégica de la empresa. Cabe destacar que se mencionó: "existe mayor apertura de contratación para estas personas en el sector público que en el privado, puesto que este último puede establecer sus propias reglas y la manera en la que se adecua a los cambios en el país"

\section{Procesos de talento humano}

Dentro de la categoría Procesos de talento humano se encontró que las labores referentes a ésta área son llevadas a cabo por profesionales diferentes a la Psicología, como abogados, contadores públicos y administradores de empresas. A partir de esto, se podría decir que existe una oportunidad de mejora dentro de estas empresas gracias a los aportes de la psicología del trabajo y las organizaciones, puesto que estos permiten humanizar los contextos laborales a partir de la adaptación y la flexibilidad con el personal de la empresa. No obstante, se encontró que los procesos se llevan a cabo dentro de la organización que eran realizados de forma superficial y algunos ejecutados por entidades externas. Sin embargo, se presenta interés por mejorar dichos procesos a fin de propiciar el bienestar del colaborador y de la empresa. Por esta razón, un punto a resaltar dentro del área de talento humano es que se busca brindar apoyo a estas personas, para que se sientan parte de la organización y puedan crecer conjuntamente con la empresa.

Los procesos que se mencionaron en las entrevistas y que poseen mayor relevancia dentro de la organización fueron: convocatoria, selección, inducción, contratación, capacitación, seguridad y salud en el trabajo, compensación y clima organizacional. Estos no han sido modificados a la luz del posconflicto, lo 
que demuestra un estancamiento en el proceso de reintegración y especialmente en la inserción laboral de estas personas.

Se manifiesta que, desde el proceso de convocatoria, debe existir inclusión social, haciendo que la oportunidad de postularse a cualquier empleo sea tangible y accesible, de modo que se eliminen barreras que lo limiten.

Por ejemplo, el proceso de selección que se lleva a cabo dentro de estas empresas es estándar, el cual se realiza para todas las personas que se presentan al cargo. Se debe cumplir con el mismo perfil requerido y atravesar todos los pasos que presenta dicho proceso, sin ningún tipo de adaptación a este tipo de problemática o población.

En cuanto a este proceso, uno de los entrevistados ve importante actualizar el currículo del colaborador, asegurarse de que no continúe en actos ilícitos, en adelante se sigue con el proceso igual que los otros trabajadores, da especial énfasis, al periodo de prueba, pues dice que este es fundamental para identificar acciones a implementar tanto en el colaborador como en el resto de personal, para garantizar una adecuada adaptación del entorno laboral.

También el proceso de inducción que se realiza dentro de las organizaciones es estandarizado para todos los trabajadores. Sin embargo, es importante adecuar este proceso, ya que es aquí donde los trabajadores se articulan a la organización, además de que se proveen de herramientas que facilitan la adaptación a su nuevo sitio de trabajo, interiorizando la cultura organizacional de la empresa.
A pesar de que no se han llevado cambios en relación a dichos procesos, sí se han realizado capacitaciones como una estrategia para sensibilizar a los trabajadores y directivos en relación a su pensamiento, actitud y actuar ante las personas en proceso de reintegración dentro de una de las empresas, a fin de propiciar la contratación de estas personas y mejorar el clima organizacional y la comunicación asertiva en el ambiente laboral. Estas capacitaciones fueron realizadas en conjunto con el área de seguridad y salud en el trabajo y con el apoyo de una profesional en la Psicología, además de personas que se encuentran involucradas y conocen del proceso de paz.

\section{Retos del área de Talento Humano}

El objetivo principal de la investigación fue reconocer los retos del área de talento humano frente a la inserción laboral de personas en proceso de reintegración en empresas del sector de construcción de la ciudad de San Juan de Pasto. Por tal razón, surge una categoría inductiva denominada Retos, la cual evidencia la importancia de realizar ajustes en los procesos del área de talento humano.

El reto principal dentro de las empresas es la transformación de la dinámica organizacional, en donde se permita adecuar a la luz del posconflicto los diferentes procesos que en ella se desarrollan, los cuales se encuentran liderados por el área de talento humano. Este último debe propiciar la integración del personal de toda la organización, para que así se desarrolle un cambio verdadero y sustancial en la cultura empresarial, su 
actitud, su pensar y su actuar. También, desde el área de talento humano se ve como un reto tomar la iniciativa de convocar, contratar, y acoger a estas personas, para ello, empoderarse de esta realidad y hacerla visible en la organización.

Hay que mencionar, además, que los procesos expuestos, al no llevarse de forma rigurosa y completa dentro de la empresa, podrían generar dificultades a largo plazo afectando a los trabajadores y la organización en general. Por ende, se deben analizar y ajustar de acuerdo a las características y necesidades de las personas en proceso de reintegración junto con el programa de seguridad y salud en el trabajo y comité de convivencia laboral, para que apoye estos procesos y sea integral su adecuación.

Asimismo, a partir de la transformación correcta y rigurosa de los procesos de selección, sensibilización frente a la contratación, inducción, clima organizacional, capacitación se podrá velar por el bienestar de estas personas dentro de la empresa. Por ejemplo, es fundamental que se realice una debida inducción y capacitación de las reglas de la empresa, acompañamiento a la adaptación a un contexto normativo, ya que, como se menciona anteriormente, los procesos que se llevan dentro de las empresas no presentan un ajuste o adaptación a este tipo de problemática o población.

Otro proceso mencionado dentro de las entrevistas fue el de clima organizacional, en donde se encontró que debe trabajarse dentro de éste para que no existan conflictos que alteren el rendimiento laboral de los trabajadores y puedan generar un ambiente de confianza en el trabajo. A su vez, en las entrevistas se enfatizó en la importancia de crear estrategias a fin de prevenir el ausentismo, pues consideran que el proceso de reintegración en el que se encuentran puede demandar tiempo de trabajo para cumplir citas o compromisos externos y se puede presentar este fenómeno.

Se visualiza la posibilidad de que la empresa genere estrategias para brindar apoyo psicológico dentro de ésta, puesto que se podrían presentar situaciones que alteren el bienestar personal y social de dicha población, además de un seguimiento para poder ver el proceso y el crecimiento de estas personas en las diferentes labores que se lleven dentro de la organización.

Por último, el mayor reto para el área de talento humano y de la organización en general es generar diferentes estrategias para sensibilizar a la población a partir de los valores corporativos y sus lineamientos, ya que sí existen grandes prejuicios y estigmas ante las personas en proceso de reintegración, causando alteraciones en el clima organizacional en los trabajadores y los directivos, puesto que se parte de una idea errónea de esta población y su historia manteniendo las barreras e imposibilitando las oportunidades laborales. Así como manifestó uno de los jefes de talento humano: "Se debe tener en cuenta que la guerra, marcó y sigue marcando la historia de Colombia, si decidimos como ciudadanos el cambio no sólo se trata de un cese al fuego, si no también y hay que brindar oportunidades laborales, socioeconómicas, educativas, culturales, que hagan en un futuro una ciudad incluyente, donde se afronten los retos y eliminen barreras". 


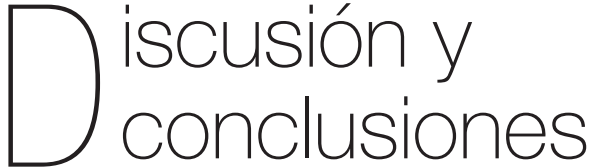

Partiendo de la idea que la empresa se encuentra conformada por personas y para personas y se reconoce como parte importante de la sociedad, esta no puede mostrarse ajena ante las problemáticas que la aquejan. Por ello, la responsabilidad social de la empresa está comprometida a aportar a solución de los diferentes problemas del país.

La desmovilización y la reintegración de las personas que han abandonado las armas trae consigo cambios sustanciales en la filosofía y la cultura organizacional. Barrios (2016) da énfasis en el papel que deben cumplir las organizaciones en el escenario del posconflicto colombiano y concluye que las organizaciones empresariales influyen directamente en el funcionamiento del país; de ahí que es vital su importancia en el posconflicto, trabajando de la mano del gobierno en el desarrollo y ejecución de procesos que garanticen la inserción al mundo laboral de los actores del postconflicto.

Dentro de la inserción laboral se vislumbra la posibilidad que tiene la población para acceder a una oportunidad de trabajo formal o informal y que le permita solventar las necesidades fundamentales como alimentación, vivienda, entre otras, y las implicaciones que tiene influir y participar dentro del tejido social del país, mejorando sus condiciones de vida y contribuyendo con el mejoramiento de la calidad en el desarrollo de la organización (Jiménez, 2013). Por esto, es pertinente manifestar que la construcción de paz y el posconflicto deben garantizar que las personas en proceso de reintegración sean parte de estas acciones, pero antes de acceder a las organizaciones, éstas deben adecuar su estructura, misión, objetivos y cultura, etc., y preparar a los trabajadores ante el cambio.

En contraste, se afirma que el acceso a un empleo estable, la búsqueda de una vida cómoda y otros beneficios materiales, actúan como motivadores para la reincidencia de personas en proceso de reintegración, pues como se expuso anteriormente, la rentabilidad que entrega el negocio del narcotráfico a esta organización hizo que se mantenga en la ilegalidad por muchos años, lo que conduce a pensar que si el acceso a dichos motivantes se ve frustrado, es probable que las personas en proceso de reintegración regresen acciones ilegales con mayores beneficios a corto plazo.

Aunado a lo anterior, en los resultados se mostró que la inserción laboral contribuye a la vida de las personas en proceso de reintegración, puesto que esto les permitirá reconstruir su vida y no volver a sus antiguas actividades. Asimismo, Barreto (2014) manifiesta que contar con un empleo, tener expectativas acerca de éste, cumplir con labores y funciones, lograr una satisfacción laboral y mantener relaciones interpersonales adecuadas dentro del trabajo, permite reconstruir y fortalecer sus recursos como persona dentro y fuera de la empresa. A estas posturas se suma J. Stiglitz, comunicación personal 31 de agosto de 2017, quien, al hablar específicamente del sector de construcción, afirma que la inserción del colaborador en este sector hace que en el país se supla una de las necesidades básicas como la vivienda. Por ende, asegura que, 
si se vive mejor, se trabaja mejor, y eso ofrece beneficios para todo el sistema.

En este sentido, se considera importante que las empresas replanteen su postura frente al aporte entre colaborador y organización, pues en algunos casos se piensa que ésta se limita en la contraprestación de servicios, es decir, el colaborador cumple con funciones establecidas y la empresa proporciona un salario. Sin embargo, como se ha evidenciado, esta relación es bidireccional y va más allá de solo eso, pues se cuenta con un personal dotado de capacidades que se pueden potenciar, como, por ejemplo, brindar aportes a la sensibilización de otros colaboradores frente al proceso de paz. Desde la importancia de la inserción laboral, se resalta la disposición de las empresas del sector de construcción estudiadas de brindar apertura para la inserción laboral de personas en proceso de reintegración, pues consideran que el trabajo permite la consolidación de paz, confirmando lo antes mencionado.

Ahora bien, las empresas no solamente deben estar dispuestas a recibir a esta población sino también a realizar modificaciones en su estructura interna para así brindar condiciones óptimas en donde se pueda crecer personal y laboralmente, como también aportar y retribuir a la empresa, y de esta manera, a la sociedad.

Es entonces, donde se ve involucrado y fundamental el aporte de la Psicología del Trabajo y las Organizaciones, contribuyendo al proceso de reintegración desde los diferentes ejes de la disciplina, reconociendo factores de riesgo que contribuyan a una inserción laboral exitosa, estableciendo entornos y redes protectoras que faciliten la incorporación y mantenimiento en un ambiente nuevo, entre otras.

Por otra parte, uno de los retos que presenta el área de talento humano es la lucha contra los estigmas presentes para esta población, que incrementan las barreras y la discriminación. Esto ha estancado algunos procesos de inserción laboral ya que los directivos, los trabajadores y muchos de los ciudadanos no se han capacitado para darle apertura a estos cambios. Lo cual, se ve argumentado por Roldán (2013), ya que sostiene que el estigma y los prejuicios para los desmovilizados aún se encuentran muy marcados en la sociedad colombiana y todavía existen muros muy altos para su reintegración social. Sin embargo, la problemática comienza con la indiferencia y la pasividad del Estado y la comunidad al no gestar mecanismos y estrategias para que la situación sea diferente.

Esta problemática no es ajena a la postura estudiada, pues los jefes de talento humano plantean posibles dificultades en el clima organizacional, ya que sus colaboradores puede que aún no acepten, ni se adecuan a los cambios realizados por el gobierno en relación al acuerdo de paz, de ahí que se genere algún tipo de discriminación, afectando el clima organizacional, las relaciones interpersonales, la integración, la satisfacción laboral y el desempeño en la empresa.

Otro punto desde el que se amerita la presencia de la Psicología en el liderazgo de procesos de talento humano frente a la inserción laboral de personas en proceso de reintegración, es al trabajar en los factores psicológicos que influyen en la adaptación laboral de esta población, 
pues es importante considerar el sufrimiento desde lo individual hasta lo colectivo en las sociedades, ya que haber agredido a personas tanto física como psicológicamente, desencadenan en dificultades en la interacción, alteraciones en el estado de ánimo, sentimientos de inseguridad, estrés postraumático, entre otras que modifican las condiciones de vida, y establecimiento de relaciones personales. Realidad que insta a los jefes de talento humano a implementar acciones encaminadas a la búsqueda de bienestar de sus colaboradores, y la labor para identificar y poner en marcha los protocolos y rutas de seguridad y salud en el trabajo que permitan aportar en la salud integral de estas personas.

Dadas las presentes condiciones y necesidades, se encuentra como dificultad que los procesos de talento humano en las entrevistas realizadas estén a cargo de profesionales ajenos a la profesión de la Psicología, hecho que lleva a que los procesos actuales se presenten de forma superficial; y en el caso de adelantar contratación hacia personas en proceso de reintegración, se podría predecir que los cambios en la empresa que se mencionan antes necesarios, se verían afectados en su implementación o desarrollo. Sin embargo, se presenta interés por mejorar dichos procesos a fin de propiciar el bienestar del trabajador y de la empresa. De ahí que se reafirma la necesidad de la psicología en las organizaciones, tanto privadas como públicas, destacando el rol y aporte sustancial del psicólogo en procesos de talento humano, y de los que de este se desplieguen.

Al mencionar al sector público, se trae a colación lo encontrado en los resultados, donde se manifiesta que existe mayor apertura de contratación en el país en este sector, el cual está conformado por organismos, empresas e instituciones pertenecientes al Estado. El sector privado, al no pertenecer al Estado y ser independiente a éste, tiene un rol diferente dentro de la sociedad. En contraste con esto, Cabrera y Niño (2016) exponen que la responsabilidad de los sectores de la sociedad colombiana es bastante grande ya que le pueden aportar al crecimiento de estas personas y del país. En ese sentido, ellos resaltan el aporte del sector privado, no sólo como un actor económico, sino que también es un actor social, ya que se reconoce la influencia de sus acciones en los demás ámbitos como político, social, económico y ambiental. Además, como lo menciona la FIP (2017), el sector privado cuenta con capacidades técnicas, administrativas, organizacionales, financieras y capital humano para contribuir con la inserción laboral de personas en proceso de reintegración.

De esta manera se concluye que la empresa en Colombia, desde cualquier sector que se posicione está íntimamente involucrada en la construcción de paz, y en ella se destaca el interés para la contratación de personas en proceso de reintegración. Sin embargo, en su estructura se encuentra la necesidad de la psicología de las organizaciones y el trabajo, pues éstas, independientemente de su accionar están llamadas a humanizar sus entornos laborales, se encuentren o no involucradas en la contratación de personas en proceso de reintegración.

Para finalizar, la presente investigación logró cumplir con los objetivos planteados anteriormente, reconociendo los retos del área de talento humano frente a la inserción laboral de personas en proceso 
de reintegración, además de que dio respuesta a una problemática latente dentro del país, permitiendo la apertura a futuras intervenciones en el área de talento humano. Aunado a lo anterior, dicho estudio da cuenta de la gran labor que conlleva el proceso de paz en la disciplina de la Psicología Organizacional, puesto que a partir de un rol activo podrán generar acciones encaminadas a mejorar los procesos del área de talento humano y humanizar el sitio de trabajo, no solo beneficiando a la empresa, sino a cada una de las personas que se encuentran inmersas allí y por lo tanto, a su círculo social cercano y a la sociedad en general. Asimismo, el aporte de la Psicología es sustancial, puesto que permite flexibilizar y adaptar los procesos de talento humano a la población con la que se está trabajando, lo que permitirá generar un vínculo con el trabajador, la empresa y la sociedad.

Cabe destacar que las categorías que surgieron en el análisis dan pie a futuras investigaciones en el tema, en donde se sugiere ampliar la muestra a investigar, así como también, enfocada a otros sectores laborales.

Antes de dar por concluido este trabajo, queremos expresar nuestro agradecimiento; a la Universidad de Nariño, por facilitar el desarrollo del presente, extendido desde el alma mater, el equipo docente y estudiantil que de forma directa e indirecta contribuyeron a los objetivos propuestos. Es importante resaltar el valioso aporte de las expertas en investigación cualitativa, y en trabajo con población en proceso de reintegración, quienes hicieron posible la construcción de la entrevista empleada. También es importante reconocer la contribución brindada desde las empresas del sector de construcción de la ciudad de Pasto, por la disposición de su personal e información que fueron la clave para los presentes resultados.

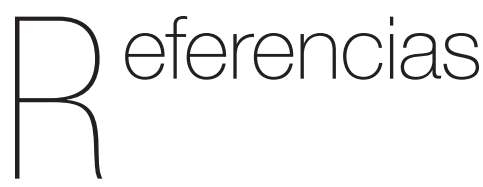

Agencia Colombiana para la Reintegración ACR (2014). Evolución del proceso de Reintegración. Recuperado el 23 de marzo de 2017 de http://www.reintegracion.gov. $\mathrm{co}$

Agencia Colombiana para la Reintegración ACR (2016). Estado de unidades de negocio. Recuperado el 22 de marzo de 2017 de http://www.reintegracion.gov.co/ es/la-reintegracion/Paginas/cifras.aspx

Agencia Colombiana para la Reintegración ACR (2016). Evolución del proceso de Reintegración. Recuperado el 23 de marzo 2017 de http://www.reintegracion.gov.co

Barreto, M. (2014). Preparar el post-conflicto en Colombia desde los programas de desarrollo y paz: Retos y lecciones aprendidas para la cooperación internacional y las empresas. Revista de Relaciones Internacionales, Estrategia y Seguridad, 9(1), 179-197. Recuperado de http://www.redalyc.org/articulo. oa?id=92731211008

Barrios, R. (2016). Responsabilidad social empresarial: el papel de las empresas en el postconflicto colombiano. Ensayos: Revista de Estudiantes de Administración de Empresas, 9(1) 45- 56.

Cabrera, D., \&Niño, L. (2016). La corresponsabilidad: el rol del sector 
privado en el proceso de reintegración. La Agencia Colombiana para la Reintegración a la vanguardia del postconflicto, 64-70

Fundación Ideas para la Paz (FIP) (2017). Aporte empresarial a la paz y desarrollo sostenible, desafíos y oportunidades. Recuperado el 14 de Enero de http:// cdn.ideaspaz.org/media/website/ document/59bc39a7dcf54.pdf

Galeano, M. E. (2003). Diseño de proyectos en la investigación cualitativa. Medellín, Colombia: Fondo Editorial Universidad EAFIT

García, A. (2017). Colombia una economía que lucha por su recuperación. Recuperado el 14 de Enero de http://www.elpais.com.co/ economia

García, M. (2011). Clima organizacional y su diagnóstico: una aproximación conceptual. Cuadernos de administración, 25(42), 43-61.

Pérez, J. G. (1999). El proceso de investigación cualitativa desde el enfoque interpretativo y de la investigación-acción. En Modelos de análisis de la investigación educativa (pp. 11-59). España: Ediciones Alfar.

Hernández, R., Fernández, C., \& Baptista, P. (2003). Metodología de la investigación. La Habana: Editorial Félix Varela.

Hernández, G. C., Valencia, J. C. N., \& Giraldo, C. M. Á. (2010). Gestión humana en la empresa colombiana: sus características, retos y aportes. Una aproximación a un sistema integral. Cuadernos de Administración, 23(41), 13-36.

Jiménez, A. J. R. (2013). Inserción laboral de la población desplazada por el conflicto armado en la frontera Colombo Ecuatoriana. Semestre Económico Universidad de Medellín, 16(34), 93-121.

Stiglitz, J. (2017). Entrevista con Santiago La Rotta. Las oportunidades económicas de la paz según Joseph Stiglitz. Recuperado de https://colombia2020.elespectador. com/economia/

López, W., Silva L., Castro, P., \& Caicedo, A. (2016). Actitudes implícitas de estudiantes universitarios frente al perdón en el marco del conflicto armado colombiano. Pensamiento Psicológico, 14(2), 49-62.

Martínez, N. B., Ocampo, A. B., Betancur, S. C., \& Bonilla, S. R. (2015). Retos de las organizaciones privadas en el postconflicto colombiano. Ensayos: Revista de los Estudiantes de Administración de Empresas, 8(8), 228-239.

Matabanchoy, S. (2012). Salud en el trabajo. Universidad y Salud, 15(1), 87-102.

Mejía, A., Bravo, M., \&Montoya, A. (2013). El factor del talento humano en las organizaciones. Ingeniería Industrial, 34(1), 2-11.

Moreno, F., \& Godoy, E. (2012). El talento humano: Un capital intangible que otorga valor en las organizaciones (human talent: An intangible capital that gives value in organizations). Daena: International Journal of Good Conscience, 7(1), 57-67.

Naranjo, O. (2005). Gestión del talento humano en la micro, pequeña y mediana empresa vinculada al programa Expopyme de la Universidad del Norte en los sectores de confecciones y alimentos. Pensamiento \& Gestión, 18, 103-137. 
Páez, F. (2015). Reinserción laboral en posconflicto: Desafíos para la Universidad y las áreas de Recursos Humanos en Colombia. Recuperado el 5 de abril de 2015 de https://revistaeducacionvirtual. com/archives/1883.

Prieto, P. G. (2013). Gestión del talento humano como estrategia para retención del personal (Tesis de Especialización), Universidad de Medellín.

Roldán, L. (2013). La inclusión laboral de los desmovilizados del conflicto en Colombia: Mecanismo emancipador de la violencia en Colombia. Universitas Estudiantes, 10(10),107-123.

Rosero, N. \& Marcos, J. M. (2015). La gestión del talento humano en las empresas colombianas una forma de hacerle frente al fenómeno de la globalización económica (Tesis de Pregrado), Universidad Militar Nueva Granada, Bogotá.

Socha, D., Lozano, K., Lozano, M., Guzmán, K., Torres, B., Díaz, D, Duran, Y., Salamanca, P., \& Mera, S. (2016). Voces rurales y urbanas del Conflicto Armado, la Violencia y Paz en Colombia. Informes Psicológicos, 16(1), 65-84. http://dx.doi. org/10.18566/ infpsicv16n1a04

Vargas, C. (2016). Las empresas de seguridad privada como fuentes de empleo para los desmovilizados en el posconflicto colombiano (Tesis de Especialización), Universidad Militar Nueva Granada, Bogotá. 